Research Article

\title{
Access Control Points: Reducing a Possible Blast Impact by Meandering
}

\author{
Martin Larcher (D), Georgios Valsamos, and Vasilis Karlos \\ European Commission, Joint Research Centre, Ispra, Italy \\ Correspondence should be addressed to Martin Larcher; martin.larcher@ec.europa.eu
}

Received 24 August 2017; Accepted 7 December 2017; Published 11 February 2018

Academic Editor: Filipe Santos

Copyright (c) 2018 Martin Larcher et al. This is an open access article distributed under the Creative Commons Attribution License, which permits unrestricted use, distribution, and reproduction in any medium, provided the original work is properly cited.

\begin{abstract}
In response to the heightened terror threat in recent years, there is an increasing interest in the introduction of access control zones at sites that are characterized by an increased likelihood of being the target of a terrorist attack, as latest data reveal that unprotected areas of mass congregation of people have become attractive to terrorist groups. Such control zones could be located within the building that has to be protected or attached to it. The elevated security needs for these areas call for a design that will consider the risk of internal explosive events. The purpose of this article is to outline a strategy for limiting the consequences of an internal blast, while guaranteeing that the produced blast wave does not propagate into vulnerable areas. In particular, the focus is on the introduction of a protective wall system in the form of a meander that allows unobstructed access of the public and at the same time reduces the possible blast inflow to the building's interior. The performed numerical simulations show that the proposed strategy yields much smaller injury risk areas compared to a design without the addition of protective walls and is recommended for upgrading the security of buildings.
\end{abstract}

\section{Introduction}

1.1. Motivation. Several trends in modern terrorism need to be considered for developing an effective protection strategy against a possible attack. In the past, the focus was on the protection of critical infrastructures. However, recent terrorist attacks shifted towards soft targets due to the increased likelihood of success without the need for careful planning, big resources, and special training of the perpetrators. Soft targets are, in general, defined as unprotected areas with high concentration of people, like shopping malls, stadia, hotels, universities, urban city centres, festivals, fairs, places of worship, train and subway stations, or Christmas markets. Such targets might consist only of the attending public (e.g., markets, open-air fairs, parks, festivals, and parades) or could also entail structural components (e.g., concert halls, theatres, museums, churches, and transportation terminals). In the recent past, Jihadist perpetrators have chosen this type of targets with the intent to cause mass casualties and due to the high social, political, and/or economic impact of a successful attack. As can be seen in Figure 1, all major attacks (involving fatalities) in Europe over the last years targeted soft targets, which is also attributed to their increased vulnerability.

It was noticed that these attacks were often performed by utilizing unconventional and/or "low-tech" weapons, such as improvised explosive devices (IEDs), knives, axes, cars, and trucks. In particular, for the attacks in Brussels (03/2016) and Paris (11/2015), IEDs were used as modus operandi. For instance, physical protection against vehicle-ramming attacks can be achieved by a properly designed barrier system. However, the detection of smaller weapons and explosives by specialized devices [1] is fundamental for reducing the possibility of an attack. The adoption of detection measures might be advisable for places of mass congregation of people but is crucial for controlling the access at high-risk sites. Moreover, the location of this detection zone is of great importance, since it is an area characterized by an increased likelihood of terrorist attacks (by using explosives and/or firearms). The present article concentrates on the design of such access control points and, in particular, their design in relation to possible attacks by the use of explosives. 

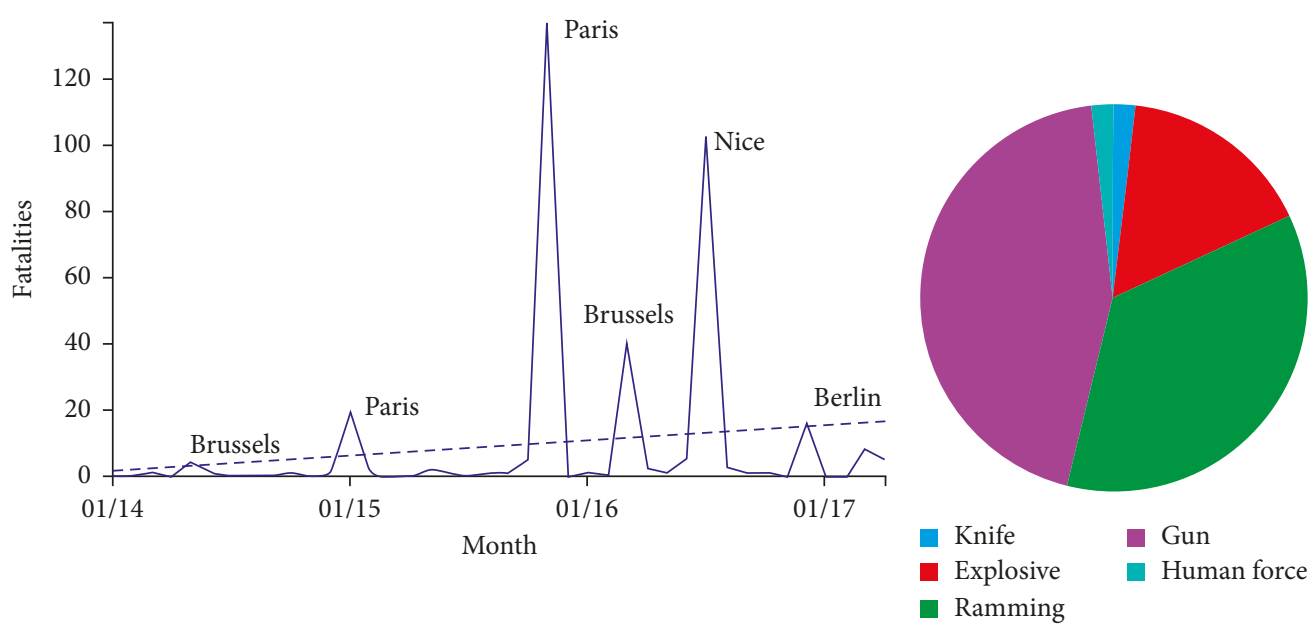

Figure 1: Fatalities due to terrorist attacks in the European Union 2014-2017, modus operandi.

Concerning the physical resistance or structural robustness of a building, a progressive collapse mechanism [2] must be avoided and glass surfaces, like windows or façades [3], should be protected against the impact of blast waves or projectiles. The physical protection of crowded places against explosive events can be performed by proper shadowing techniques, for example, by using concrete blocks [4].

1.2. Risk Mitigation. The creation of an efficient plan for the protection of critical infrastructures and soft targets starts with identifying the potential risk. To ascertain the risk of an attack, the analyst has to weigh its probable consequences, the most suitable tactic, and the weaknesses that may be exploited by the aggressors in their effort to impose damage and casualties. This means that for calculating the potential risk, the combination of threat level, vulnerability level, and target value is required. The threat level can be defined as the probability of occurrence of an attack in a specific period of time, causing abnormal loading to the structure. The vulnerability level is linked to the identification of security weaknesses and the potential damage to the system resulting from an event with a given intensity. The target value can be quantified with respect to the damage in the target's assets, such as injuries of the occupants, human life loss, capital value, reconstruction cost, and disruption of services and functions.

A carefully designed protection strategy and crisis management plan can effectively reduce the impact of an attack to the target's asset value. The implementation of a detection and investigation scheme can deter possible aggressors and decrease the threat level, while the assessed vulnerability can be reduced by strengthening the examined structure to limit the consequences of an attack.

Many buildings can be identified as potential targets of a terrorist attack. Accessibility to these buildings is often restricted, and people entering the premises (in particular external visitors) are subjected to security checks to reduce the likelihood of an attack at the building's interior. Even though an access control strategy does not guarantee detection of all possible threats, it can certainly be assumed that perpetrators cannot enter carrying big amounts of explosives or firearms. Practically, this means that both the risk of possible attacks inside the building and their consequences are reduced.

The location of a security control zone, where people are checked for the presence of explosive materials and weapons prior entering to the building, should be carefully selected. Prior experience from military applications shows that a dedicated building (guardhouse), detached from an existing structure, reduces possible consequences in case of an attack at its interior. Such a design approach is feasible if space is available, but often in the densely built urban environment buildings occupying nearly all the available lot and construction of a detached structure is not an option. In the current article, measures for the protection of access control areas in closed environment against explosions are presented. In particular, the proposed strategy is based on the employment of a meandering wall to reduce the possible inflow of the produced blast wave to a building's interior.

1.3. Policy Context. Security is one of the recent priorities of the European Commission. The European Agenda on Security clearly advocates that the protection of critical infrastructures and soft targets presents a real challenge. The main objective is to gather best practise principles and produce guidance for mitigating the risk of terrorism. In particular, it is highlighted that the protection of soft targets and human life is of utmost importance and additional efforts are required from both state and private security stakeholders.

\section{Explosion Effects}

2.1. Blast Loading. Blast waves are typically characterized by a compression phase (positive phase) with a very high peak overpressure, followed by an underpressure phase (negative phase). Figure 2 shows an idealized profile of the pressure in relation to time for the case of a blast wave and presents some relevant parameters. The compression phase includes an almost instantaneous increase from the ambient pressure $\left(p_{0}\right)$ to a peak pressure $\left(p_{0}+p_{\max }\right)$ at the arrival time $t_{a}$, when the 


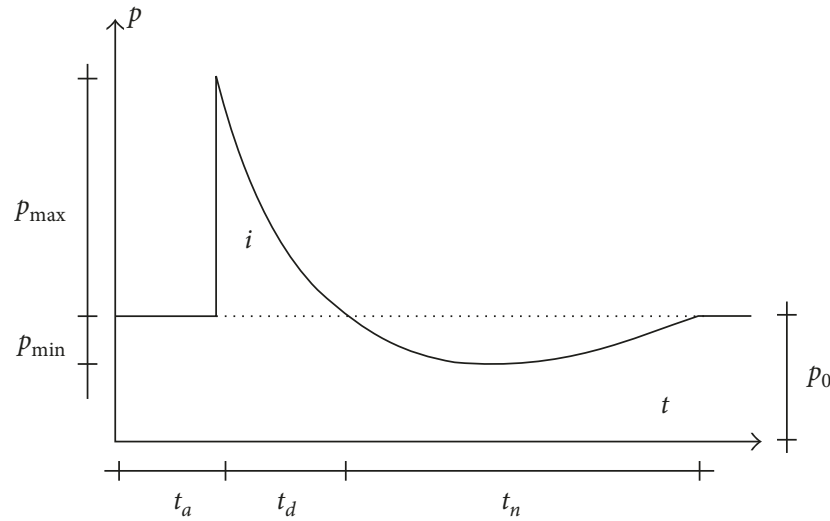

FIGURe 2: Pressure-time history for an ideal free-field air-blast wave.

shock front reaches the point of interest. The time required for the pressure to reach its peak value is very small, and for design purposes, it is usually considered as equal to zero. After its peak value, the pressure decreases at an exponential rate until it reaches the ambient value at $t_{a}+t_{d}, t_{d}$ being called the positive phase duration. After the positive phase, the pressure drops below the ambient value, attaining its minimum value $p_{\text {min }}$, before finally returning to it after $t_{n}$ (negative phase duration). The positive impulse $i$ is defined as the integral of the overpressure curve over time and is distinguished in positive and negative according to the relevant phase of the blast wave time history. More detailed information on the blast wave parameters is provided in [5-8].

The loading conditions of an externally loaded structure can be distinguished into three types: impulsive, dynamic, and quasi-static loading (Figure 3). Loads with very short duration (relative to the structure's or component's fundamental natural period) are known as impulsive loads, for example, near-field explosions of smaller charges (rucksack bombs). The structural consequences of such explosions range from shear-type failures (e.g., of fragile parts like window panes) to bending-type failures (e.g., stiffer concrete structures). Explosions with very small distances or even contact detonations could cause even spalling and scabbing [9]. If the duration of the excitation is longer than the natural period of the structure or of the component (dynamic loading), the predominant structural failure mode is bending as in the case of far-field explosions (car bombs) and gas explosions. A static load simulation approach can be used only if the examined pressure increases at a very low rate (quasi-static loading). For the other two loading conditions, sophisticated numerical models should be utilized, for example, an explicit finite element analysis. For a particular structure and load type (e.g., pressure), these loading regimes can be schematically presented by an iso-response curve in a so-called PI (pressure-impulse) diagram (Figure 3). If a probabilistic approach is of interest, the probability of damage could also be inserted in the diagram (e.g., Figure 4).

2.2. Venting/Pressure Release. The diagram shown in Figure 2 is only valid for spherical or hemispherical, free air, or

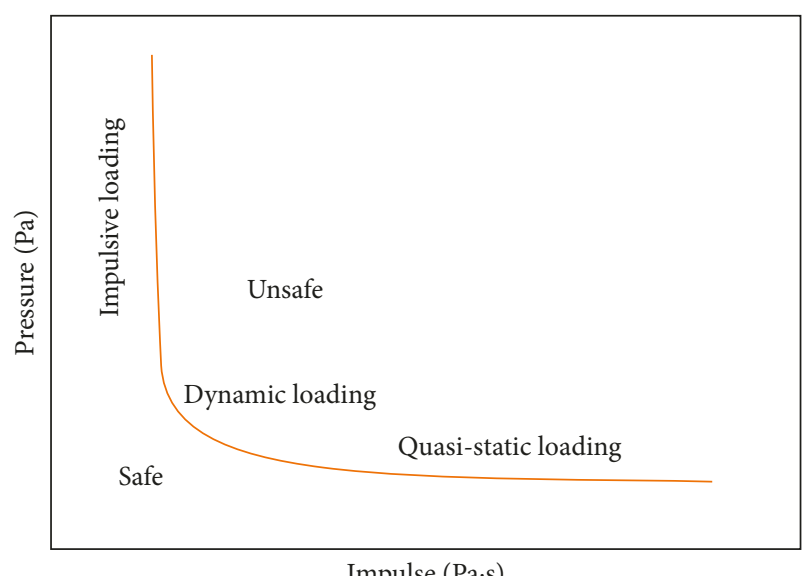

Impulse (Pa-s)

Figure 3: Typical PI diagram: impulsive, dynamic, and quasi-static loading (logarithmic scale).

surface bursts, respectively. Reflections, shadowing, or channelling phenomena can significantly alter the behaviour of a blast wave. In such cases, the calculation of the blast wave parameters with the empirical and semiempirical formulas proposed in [5] could result in significant errors. Thus, the use of numerical fluid-structure interaction calculations can provide a detailed identification of the loading time history of a structure or a component.

Internal explosions in confined rooms or bunkers where the pressure wave cannot escape result in a static remaining pressure after the explosion. Certain concepts for designing venting surfaces for gas explosions (e.g., $[10,11])$ are publically available. However, blasts from solid explosives in confined spaces are in general faster and are characterized by much higher peak pressure and often smaller impulse values in comparison to gas explosions. The remaining static pressure from such explosions is significant and must be considered during design. Release surfaces might prove effective [12], as they are able to reduce the remaining pressure after an explosion even though their effects are not immediately evident. Nevertheless, venting surfaces cannot reduce the peak pressure associated with the first arrival of the blast wave.

2.3. Afterburning. The afterburning phenomenon may prove significant for internal explosions, depending on the type of explosive burnable products that remain after the detonation. These hot products mix with the surrounding air and burn if the conditions (in particular oxygen availability) allow combustion. Also, the presence of aluminium powder in the explosive contributes to the combustion process which leads to an additional pressure after the first peak pressure. The effect of afterburning on the resulting pressures is influenced by many factors. In numerical simulations, the approach of Miller [13] is commonly used, who proposed the addition of a certain amount of energy to the model after a specific time. Nevertheless, afterburning is not considered in the present article since injuries are most likely associated with the initial blast wave and not the afterburning. 


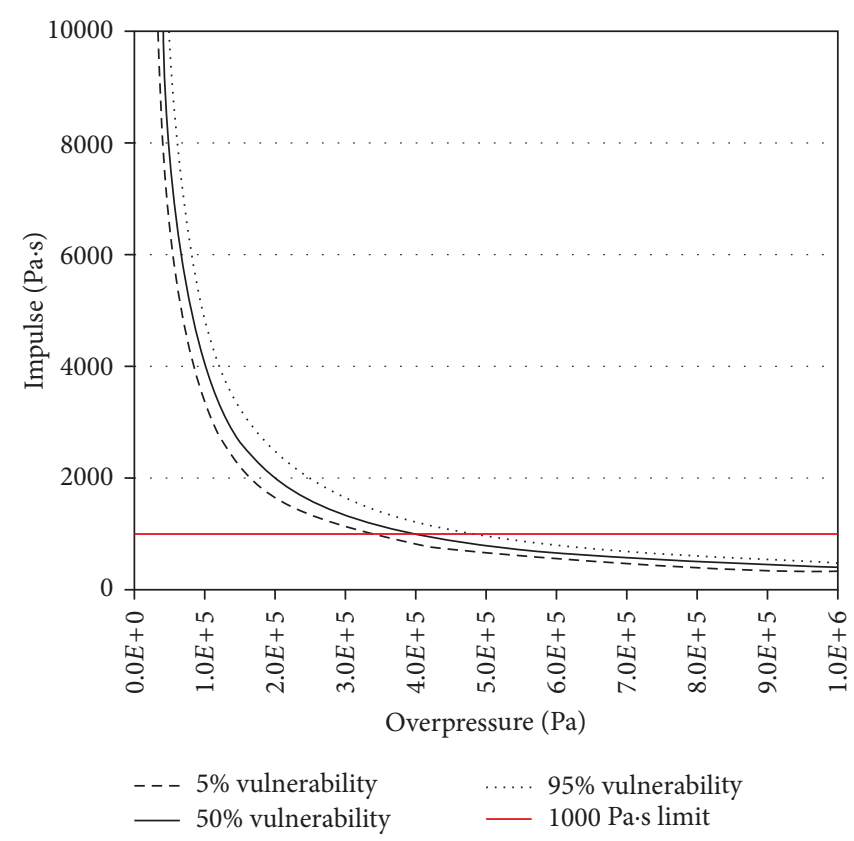

Figure 4: Vulnerability of humans (head impact).

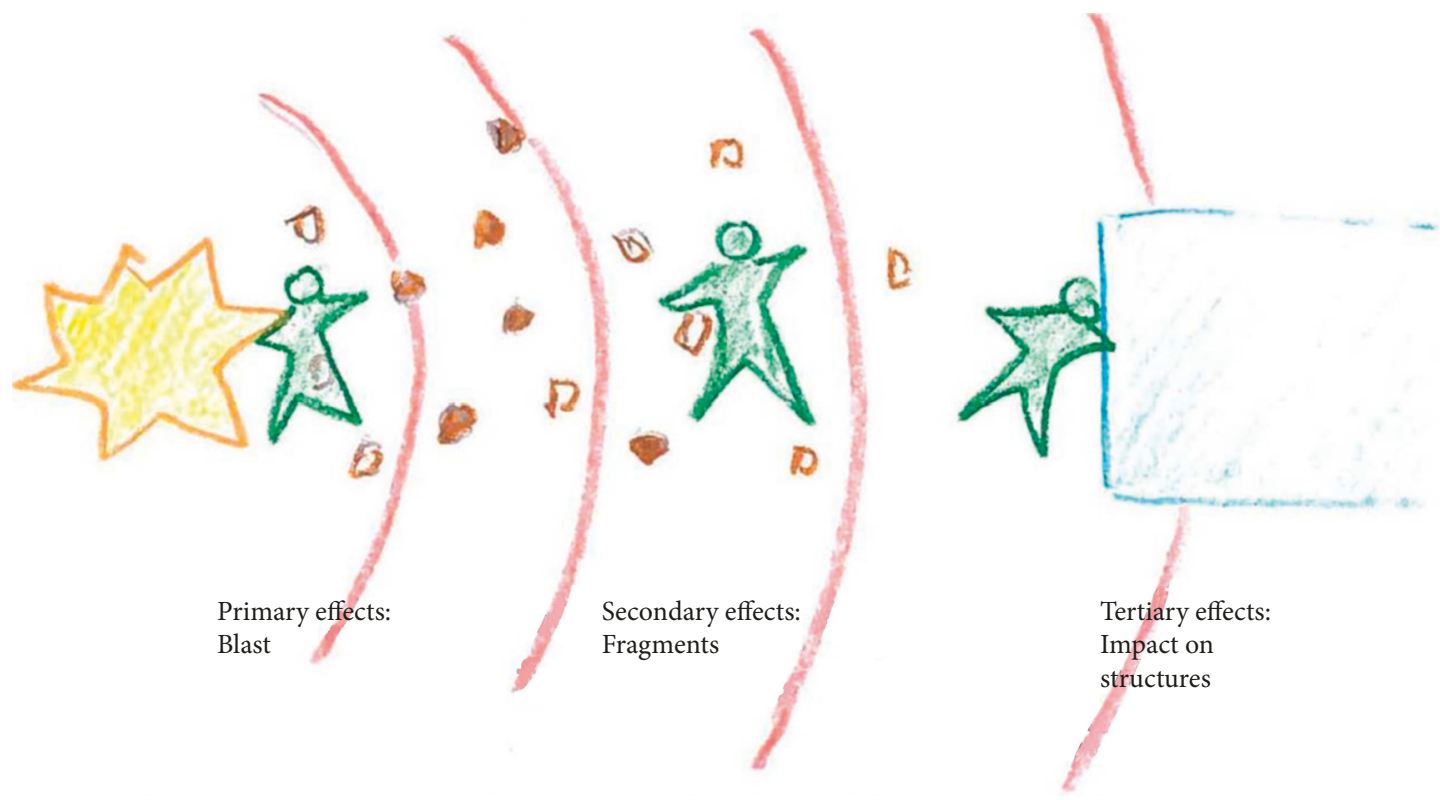

Figure 5: Blast effects of an explosive charge (yellow) on humans (green) due to the created blast wave (red) and fragmentation (brown).

2.4. Vulnerability of Humans. The effects of an explosion on human body can be distinguished into three broad categories (Figure 5). Concerning explosion-induced injuries, the risk due to the primary blast effects on humans has already been precisely described by several authors [14]. However, this is not yet the case for secondary blast effects that are related to the produced fragments either from the explosive charge or from the failure of structures that are propelled with high velocities striking the human body. A straightforward procedure for assessing the additional risk due to the presence of splinters as part of the secondary blast effects is currently under development [15].
In this work, the formulation of the human injury risk model is based on the work of González Ferradás et al. [16] and Mannan [17]. It utilizes the peak overpressure $p_{\max }$ (in Pa) and the positive impulse I (in Pa.s) to determine the probability of eardrum rupture and the probability of death.

2.4.1. Causes of Death. Regarding possible causes of death, three different cases are considered: head impact, whole body impact, and lung haemorrhage. Using a PI diagram [14], it has been proven that the head impact is the dominant cause of death and its probit function can be defined as 


$$
Y_{1}=5-8.49 \ln \left(\frac{2430}{p_{\max }}+\frac{4 \cdot 10^{8}}{P_{\max } \cdot I}\right)
$$

2.4.2. Eardrum Rupture. The probit function $Y_{4}$ of eardrum rupture is described according to the following equation:

$$
Y_{4}=-12.6+1.524 \ln \left(p_{\max }\right) \text {. }
$$

The probability of occurrence $R$ (or the percentage of the affected population) of the corresponding injury is next determined for each death-related function using the following equation from González Ferradás et al. [16], which is a good approximation of the relevant cumulative normal distribution:

$$
R_{i}=-3.25 Y_{i}^{3}+48.76 Y_{i}^{2}-206.6 Y_{i}+270.35 .
$$

Human risk is presented in Figure 4 as a PI diagram for a 5, 50, and $95 \%$ probability of death. Clearly, for calculating the risk of life loss at every point, both the peak pressure and the impulse values are required, meaning that a full fluidstructure interaction (FSI) analysis is required. It is noted that the influence of flying debris (secondary blast effects) is not included in these formulas.

These formulations are developed for the positive phase of spherical or hemispherical blast waves from free air and surface bursts, respectively, and can be used with both incident and reflected blast parameters. In case of confined explosions, the remaining static pressure results in an increased impulse, a phenomenon that is not considered from the available formulas. The duration of the explosions simulated in [16] is in general shorter than the time required for the effects of venting to be evident, and therefore, the above formulas can be used. In order to reduce the complexity, as an additional criterion, an impulse limit of $1000 \mathrm{~Pa} \cdot \mathrm{s}$ is used to identify critical zones at the building's interior. UFC 3-340-02 [18] proposes a limit of $1380-1725 \mathrm{kPa}$ for lethal damage as a result of lung haemorrhage. This value ignores the head impact, a fact that is also recognized in UFC 3-340-02 where a much lower threshold is referenced $(16 \mathrm{kPa})$. In the present study, the impulse value of $1000 \mathrm{~Pa} \cdot \mathrm{s}$ is used as an indicative limit motivated by Figure 4 and taking into account a minimum peak overpressure value of about $250 \mathrm{kPa}$. The impulse parameter is used since it is more appropriate for confined explosions. The objective of this approach is to acquire qualitative results to investigate the effects of meandering. For quantitative results, the abovementioned risk formulas should be used.

\section{Blast Protective Design}

Protective design of critical infrastructures against terrorist attacks is usually performed in several steps:

(i) The first step is establishing plausible attack scenarios, which is a joint decision of the owner or stakeholder and the designer. Assessing the probability of occurrence of a terrorist attack through estimating sources of threat could be an appropriate tool for defining and choosing possible scenarios. An indication is given, for example, by the North Atlantic Treaty Organization's (NATO) Standardization Agreement (STANAG) 2280 [19].

(ii) The next step is to calculate the corresponding loads on the structure by identifying the worst case scenario. In some cases, this can be performed by using simple formulas for the blast wave propagation, as those proposed by Kingery and Bulmash [5] and Kinney and Graham [20], or by using a series of diagrams included in Unified Facilities Criteria such as UFC 3-340-2 [18]. Bogosian et al. [21] compared these equations with experimental data and showed that in urban environments, the propagation of the blast wave is not spherical and differences appear at the calculated blast parameters. For such complicated geometries, numerical simulations may be necessary to assess with greater precision the loading history of a structure, as described in [22].

(iii) Subsequently, the protection strategy against the determined blast load according to the individual structural characteristics of the examined building (e.g., windows or facades) should be defined. Several options, validated by numerical or experimental methods, can be adopted, as described in [23].

Among the various structural and nonstructural elements, windows and facades are considered critical when facing an explosion due to their extremely fragile nature. In addition, a progressive collapse mechanism must always be prevented, as it could lead to a large death toll. In general, the explosive energy decreases rapidly by increasing the standoff distance. Hence, employing perimeter protection measures and restricting the access to a site constitute one of the most effective methods for decreasing its vulnerability. For example, protection against vehicle access can be achieved by the application of various barrier systems, as shown in [24].

3.1. Access Point Design for Building Structures. Depending on the security level of a site, access control measures may also be employed for the entrance of the public. The control zone should be placed in an area which should be resistant to both exterior and interior explosive events. Long queuing should be avoided to minimize the likelihood of the access point becoming a target of a terrorist attack. In case of a blast, flying debris from the failed parts of the structure (in particular from glass failure) may cause extensive injuries or fatalities. Therefore, incorporating venting in the form of release surfaces is important for mitigating the effects of a blast, in particular, if the access zone is inside the main building or attached to it. The release surfaces should be light, to be easily pushed aside by the propagating blast wave, or fragile, to instantly fail under the blast pressure. To prevent people's injury from the potential fragments of the failed opening, the release surfaces are often located at the roof of the structure. Nevertheless, the effects of venting should not be overestimated. The release surfaces (whose size is up to $10 \%$ of the 


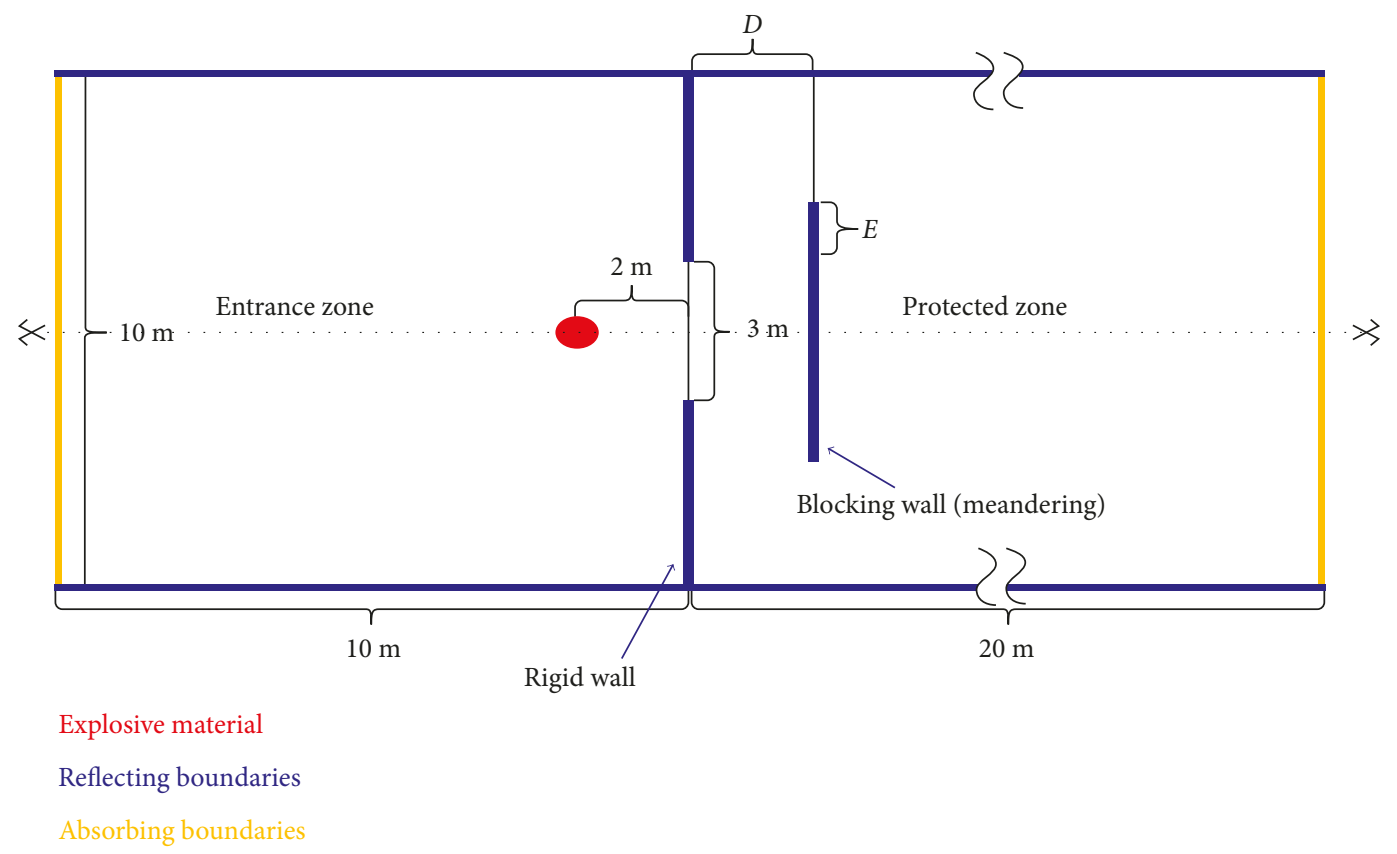

Figure 6: Geometry of the case study model.

room's size) have a very small impact on the peak pressure but are effective for reducing the remaining static pressure after the explosion. Clearly, the proper design of release surfaces is a challenging task since no clear indications exist in the open literature. Usually, it is advised to follow a design approach that depends on the geometry of the control zone, while the size of the openings ranges from $3 \%$ to $8 \%$ of the room's (control area's) size. Thus, these values serve only as an indication, since the actual release surface size depends also on its cross section and the determined blast scenario.

If the access control zone is located within or is connected to the main building, the propagation of the pressure wave into the adjacent structure should be avoided to confine the explosive effects only in one area. Constructions having very stiff or blast resistant doors are complicated, impractical, and space demanding since these doors must be duplicated. As shown below, a so-called meandering wall can achieve a similar protection level maintaining a design that favors the unobstructed flow of people.

\section{Numerical Simulations}

The current section is dedicated to the analysis of a case study simulating an explosion within an access control zone. The objective of the case study is to investigate the way a rigid (meandering) wall affects the propagation of the blast wave into an area of the building that needs to be protected. Moreover, a parametric study on the influence of the basic dimensions of the meandering wall on the attenuation of the produced detonation wave is carried out. The numerical simulations are performed with the explicit FE code EUROPLEXUS (EPX) [25], which is jointly developed by the French Commissariat à l' Energie Atomique et aux Energies Alternatives (CEA) and the Joint Research Centre of the
European Commission (JRC Ispra). EPX employs sophisticated fluid-structure interaction techniques and is suitable for simulating fast and severe loadings on structures such as impact and blast. The CAST3M code [26], also developed by CEA, was used to generate the mesh for the numerical models, while ParaView software [27] was used for postprocessing the results.

4.1. Case Study. To reduce complexity, a simple geometry has been selected for the case study. A rectangular area is separated by a rigid wall into two parts. The first compartment is considered as the entrance zone where a possible intruder can enter carrying an improvised explosive device (IED) and walk till the opening of the rigid wall that leads to the second compartment. Just before the opening that connects the two parts, a thorough control of all persons entering the second part is considered to be implemented. The second compartment is regarded a vulnerable zone that needs to be protected, as a possible blast wave can become critical for the personnel or the structural integrity of the building.

Figure 6 shows the geometrical details of the investigated area. The entrance zone, located on the left, is connected to the protected zone, on the right, through a $3 \mathrm{~m}$ wide opening. The opening's width guarantees a relatively high flow of the people to avoid queuing. The height of both zones is 3 meters, while the detonation centre is located 1.5 meters from the ground level (average value for the location of a backpack in the vertical axis) and is centred to the opening, based on a worst case scenario. This is assumed to be the closest point to the protected zone that a perpetrator can reach prior to being intercepted. The mass of the IED has been selected to be equal to 25 kilograms of TNT equivalent, as an upper limit for the amount of explosives a pedestrian could carry in a backpack or small suitcase. 
TABLE 1: Parameters for the JWL EOS for TNT (Dobratz and Crawford [31]).

\begin{tabular}{lccc}
\hline Parameters & Description & Parameters for explosive & Parameters for air \\
\hline $\mathrm{A}(\mathrm{Pa})$ & Material constant (experimental) & $3.7121 \mathrm{e} 11$ & $3.7121 \mathrm{e} 11$ \\
$\mathrm{~B}(\mathrm{~Pa})$ & Material constant (experimental) & $3.23 \mathrm{e} 9$ & $3.23 \mathrm{e} 9$ \\
$\mathrm{R} 1$ & Material constant (experimental) & 4.15 & 4.15 \\
$\mathrm{R} 2$ & Material constant (experimental) & 0.95 & 0.95 \\
$\mathrm{Beta}$ & Material constant (experimental) & 0.25 & 0.25 \\
$\mathrm{RHO}\left(\mathrm{kg} / \mathrm{m}^{3}\right)$ & Density & 1630 & 1.3 \\
EINT $(\mathrm{J} / \mathrm{kg})$ & Current internal energy & $0.21978 \mathrm{e} 6$ \\
Per unit mass & $4.29 \mathrm{e} 6$ & 0.3 \\
OMEGA & Specific heat ratio & 6930 & 0.3 \\
$\mathrm{D}(\mathrm{m} / \mathrm{s})$ & Detonation velocity & - \\
\hline
\end{tabular}

TABLe 2: Material properties for the ideal gas law for atmospheric air.

\begin{tabular}{lccc}
\hline Density $\left(\mathrm{kg} / \mathrm{m}^{3}\right)$ & Specific heat at constant volume $(\mathrm{J} / \mathrm{kg} \cdot \mathrm{K})$ & Gamma ratio & Reference pressure $(\mathrm{Pa})$ \\
\hline 1.3 & 716.75 & 1.4 & $1 \mathrm{e} 5$ \\
\hline
\end{tabular}

As shown in Figure 6, the distance $D$ between the opening and the introduced meandering wall and the dimension $E$ that refers to the overlapping between the additional blocking wall and the preexisting rigid wall are not explicitly determined. These two parameters are selected as variables in the performed parametric study in order to quantify their influence on the blast wave propagation. $D$ ranges from 1 to 3 meters, corresponding to construction specifications (e.g., wheelchair access and personnel flow). Similarly, $E$ ranges from 0.5 to 2 meters, to allow a minimum distance of 1.5 meters between each extremity of the meandering wall and the lateral walls of the building and to guarantee comfortable access to the attached protected zone.

4.2. Numerical Model. The structural parts of the building in the case study are assumed rigid, meaning that the walls, the ceiling, and the floor of the structure are nondeformable. A postanalysis investigation of the recorded maximum pressures indicated that thick, stiff structural elements like concrete or steel walls respect this basic assumption. This hypothesis results in decreased computational power demand, since there is no need to include deformable structural parts or perform cumbersome fluid-structure interaction (FSI) calculations. Moreover, the absence of structural deformations allows an accurate study of the influence of the two parameters ( $D$ and $E$ ) on the blast wave propagation.

As already mentioned, for the numerical calculations, a purely Eulerian approach is utilized, where only the fluid part of the model is considered. The Eulerian formulation considers the computational mesh fixed while the fluid (particles) moves relative to these fixed grid points. The conservation laws of mass, momentum, and energy are expressed in a spatial framework. The discretization is based on cell-centred finite volume (FV) formulations in which the governing equations are solved in integral form. This method is conservative since the formulation ensures that the flux entering a given volume is identical to that leaving the adjacent volume. The numerical fluxes between adjacent FVs were calculated using the Harten-Lax-van Leer-contact (HLLC) Reimann solver [28].

The fluid domain was modelled with $3 \mathrm{D}$ cubic finite volumes (CUVF) of 0.1 meter edge size. The size of the cubic volumes is selected after a convergence parametric study. On the extremity surfaces of the two different zones (yellow lines on Figure 6), absorbing boundaries have been introduced, in order to simulate nonreflecting atmospheric boundaries. Suitable shell elements (CL3D) that allow the introduction of an absorbing medium have been employed and from a mathematical point of view represent a supersonic outlet. As already mentioned, the rigid parts of the model are simulated as reflecting boundaries. For a $3 \mathrm{D}$ cubic FV, a free surface is considered by definition as a reflecting boundary. A simple duplication of the fluid nodes at the area where the rigid structure is located results into free FV surfaces that behave as reflecting boundaries.

The representation of the blast load is performed via the $1 \mathrm{D}$ to $3 \mathrm{D}$ mapping technique [29], during which a $1 \mathrm{D}$ numerical calculation that simulates the blast load is performed with a very fine mesh. The data obtained from the 1D calculation are mapped into a spherical shape and inserted in the coarser 3D mesh. An important detail that should be taken into account is that when transferring the results of the $1 \mathrm{D}$ analysis into the $3 \mathrm{D}$ mesh, the blast load is considered spherical, so the transformation should be performed before the wave comes to contact with any obstacles. This mapping technique can significantly accelerate the calculation time without sacrificing the accuracy of the results. For the representation of the detonation load in the $1 \mathrm{D}$ calculation, the Jones-Wilkins-Lee EOS law is used [30]. The material parameters for the TNT are proposed by [31] and are included in Table 1. The perfect gas law is employed for the representation of the atmospheric air in the fluid simulation. The material parameters for the atmospheric air are shown in Table 2. Finally, one 

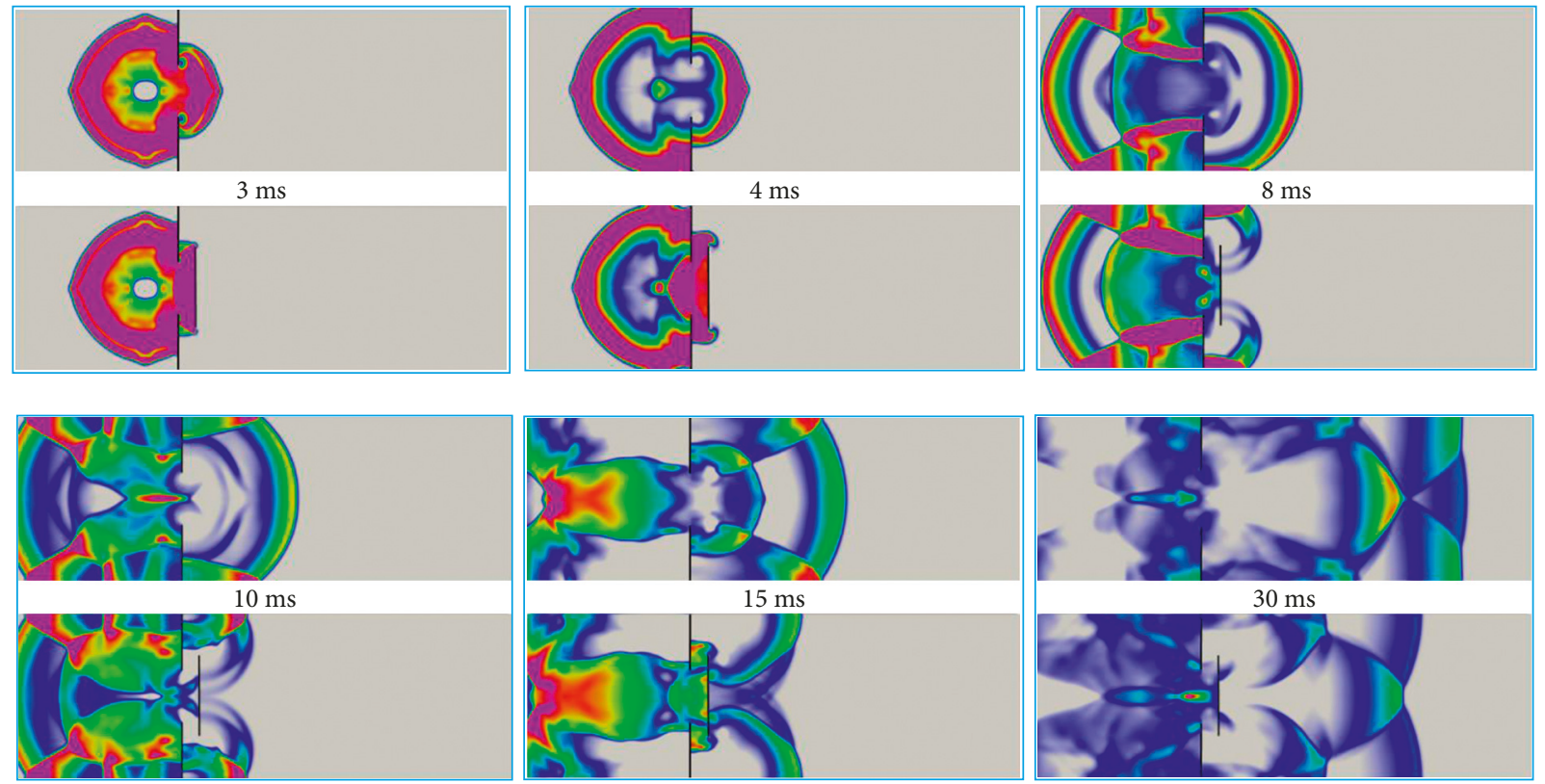

Pressure

$\begin{array}{lllll}1.0 e+05 & 1.5 e+05 & 2.0 e+05 & 2.5 e+05 & 3.0 e+05\end{array}$

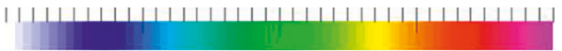

Figure 7: Pressure distribution of the blast wave for the "open door" model and a "meandering" model $(D=1 \mathrm{~m} ; E=1 \mathrm{~m})$ for several time frames $(\mathrm{Pa})$.

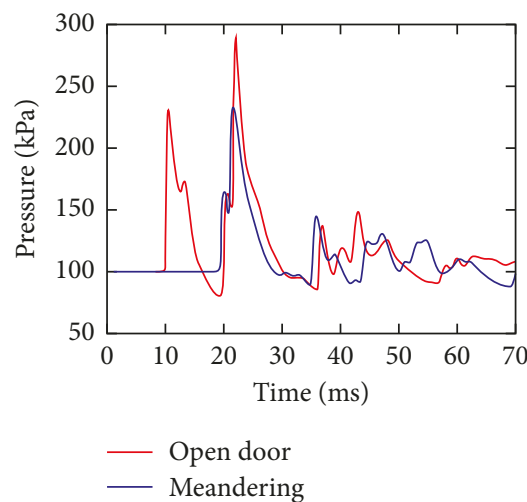

(a)

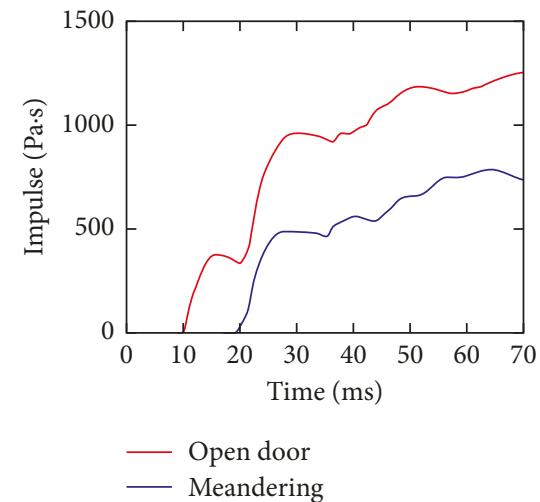

(b)

Figure 8: Comparison of (a) pressure and (b) impulse values for a point located in the protected zone for an "open door" model and a "meandering wall" model $(D=1 \mathrm{~m} ; E=1 \mathrm{~m})$.

symmetry plane was utilized by modelling only one half of the fluid mesh.

4.3. Numerical Results. The objective of the numerical investigation is to determine the effectiveness of the introduced meandering wall. Figure 7 displays the behaviour of the propagated blast wave for the "open door" case and a "meandering" case $(D=1 \mathrm{~m} ; E=1 \mathrm{~m})$ for a plane view at the height of 1.5 meters. A comparison of the two cases is performed at several time frames in order to provide a clear representation on the diversion of the blast wave from the additional wall. As already mentioned, half of the geometry was modelled in the numerical calculations so in order to present the full model in Figure 7, special mirroring techniques are used in the postprocessing phase. The first subfigure presents the pressure field distribution at $3 \mathrm{~ms}$ after the detonation. At that time frame, the wave front starts being reflected on the meandering wall, as it is clear from the differences between the two models. For the "meandering" case, the blast wave starts to enter the protected zone at $4 \mathrm{~ms}$ and is split into two smaller waves propagating from both sides of the meandering wall. At $8 \mathrm{~ms}$, it is evident that, in the "open door" case, there is one strong wave front propagating into the protected zone, while for the "meandering" case there are two weaker wave fronts. The wave propagation continues in a similar way for both cases until $30 \mathrm{~ms}$ after the detonation, where the two wave fronts of the "meandering" 


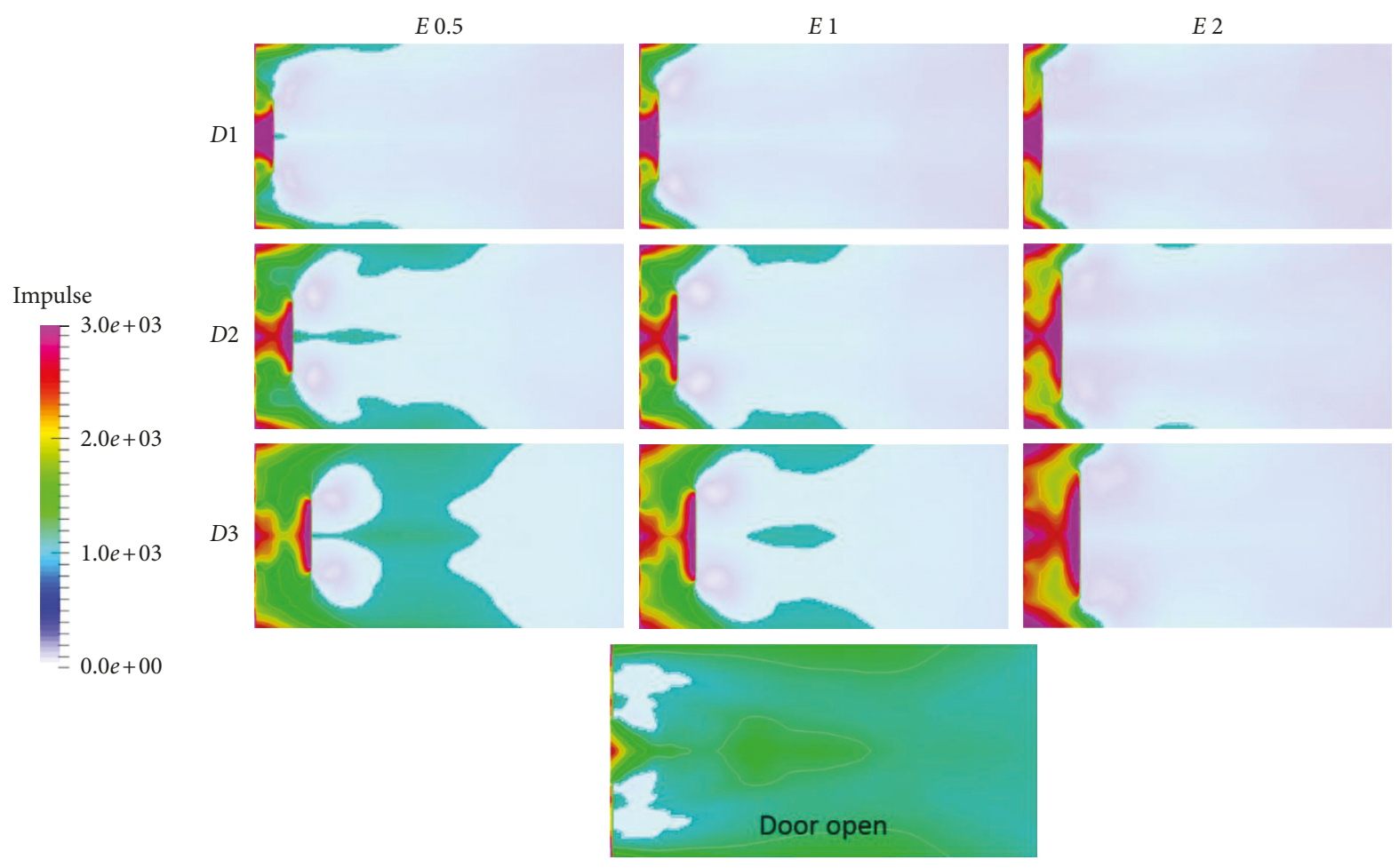

FIGURE 9: Zone of impulse higher than $1000 \mathrm{~Pa} \cdot \mathrm{s}$ for the "meandering" studied cases and the "open door" model (parameters $D$ and $E$ in $\mathrm{m}$ and impulse in pa.s).

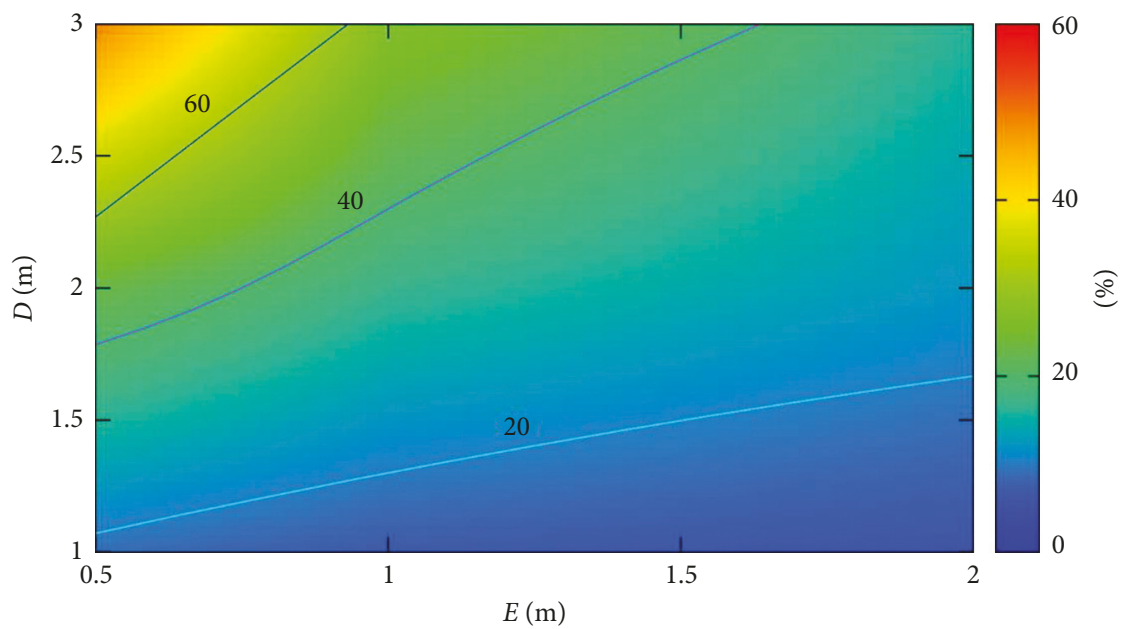

Figure 10: Percentage of affected area spectrum versus the investigated parameters for the "meandering" model.

case are united into one. But, as can be noticed, even in that phase the wave front of the "meandering" case is weaker than the one of the "open door" case.

The meandering wall reflects part of the initial blast wave and guides it back to the entrance area, resulting in a slightly stronger blast wave into the entrance zone. Since this zone has already been significantly affected from the initial blast wave, the additional energy due to the presence of the "meandering" wall does not affect the injury risk probability. Figure 8 presents the pressure and the impulse history for the abovementioned cases, at a point that is located at the middle of the protected zone, 7 meters from the opening, and 1.5 meters from the ground level. From the pressure-time history, it is clear that in the "meandering" case the first peak is eliminated, while the second peak, that results from the reflection of the wave onto the lateral walls, is lower. Similar results can be derived from comparing the impulse-time histories, as there is a significant reduction of the total impulse when the meandering wall is present.

The next figures present the influence of the dimension and location of the meandering wall on the blast wave propagation. As has already been described in previous sections, areas with an impulse value larger than $1000 \mathrm{~Pa} \cdot \mathrm{s}$ are considered as high injury risk zones. Figure 9 presents the injury risk areas for the protected zone of the examined 
building for several studied geometries. The results refer to the same horizontal cut that is presented in Figure 7, but the output quantity is the calculated impulse. Areas characterized by impulse values higher than $1000 \mathrm{~Pa} \cdot \mathrm{s}$ are highlighted. $D$ and $E$ determine the location and the size of the meandering wall as described earlier.

In the same figure, the "open door" case is also included for comparison purposes. In the "open door" case, the majority of the protected zone results in a high injury risk area. In the "meandering" case, the size of the high injury risk area varies but is always smaller than the "open door" case. It is clear that the area before the additional wall is always unprotected irrespective of the presence of the meandering wall. Moreover, in some cases, high impulse values are also recorded at the sides of the meandering wall due to the relatively strong wave fronts that are present at both those areas. Figure 10 summarizes the output of the parametric study by presenting the percentage of the injury risk area in the protected zone (above $1000 \mathrm{~Pa} \cdot \mathrm{s}$ ) versus the two examined parameters. From comparing the several "meandering" cases, it can be concluded that if $D$ is small (distance between the door and the added wall), the influence of $E$ (dimension of overlapping length) is of minor importance. However, if $D$ is large, the influence of $E$ is significant (if $E$ decreases, the blast wave that enters into the protected zone is stronger and the high injury risk areas are becoming larger).

\section{Conclusions}

Access control points that are located inside or attached to buildings are often adopted due to local constraints. Their design must consider possible terrorist attack scenarios (e.g., explosions) at the interior and exterior of these access control points. The mitigation of the consequences should such a terrorist attack materialize inside an access point and in the attached building can be achieved by using venting (release surfaces) and, in particular, meandering walls. The investigation shows that the reduction of the pressure is significant if meandering walls are used and that these walls can, therefore, play an important role in separating access control points from the attached buildings. Nevertheless, it is obvious that meandering walls can be considered an effective risk reduction measure and not as a full protection solution. The risk reduction concerns both blast wave effects and, for obvious reasons, fragments resulting from explosions and shooting attacks. Herein, the idealized situation that is investigated can be extended to include additional parameters. As has been presented in the current article, the presence of a rigid wall standing between an explosion's detonation centre and a target, can effectively reduce the severity of the impacting blast wave. It is therefore recommended the addition of such elements (varying in size and geometry) in order to mitigate the risk of terrorist attacks.

\section{Conflicts of Interest}

The authors declare that there are no conflicts of interest regarding the publication of this paper.

\section{References}

[1] A. M. Lewis, "State of the art report on European legislation relating to explosives and explosive detection system for nonaviation configurations," Tech. Rep. JRC84076, Joint Research Centre, Ispra, Italy, 2013.

[2] B. M. Luccioni, R. D. Ambrosini, and R. F. Danesi, "Analysis of building collapse under blast loads," Engineering Structures, vol. 26, no. 1, pp. 63-71, 2004.

[3] M. Larcher, M. Arrigoni, C. Bedon et al., "Design of blastloaded glazing windows and facades: a review of essential requirements towards standardization," Advances in Civil Engineering, vol. 2016, Article ID 2604232, 14 pages, 2016.

[4] R. Hajek and M. Foglar, "Numerical and experimental analysis of the effect of rigid barriers on blast wave propagation," Journal of Structural Engineering, vol. 141, no. 12, p. $04015061,2015$.

[5] C. N. Kingery and G. Bulmash, "Airblast parameters from TNT spherical air burst and hemispherical surface burst," Tech. Rep. ARBRL-TR-02555, Defence Technical Information Center, Ballistic Research Laboratory, Aberdeen Proving Ground, MD, USA, Tech. Rep. ARBRL-TR-02555, 1984.

[6] M. Larcher, "Pressure-time functions for the description of air blast waves," Tech. Rep. JRC46829, Joint Research Centre, Ispra, Italy, 2008.

[7] V. Karlos and G. Solomos, "Calculation of blast loads for application to structural components," Tech. Rep. EUR 26456EN, Joint Research Centre, Publications Office of the European Union, Luxembourg, 2013.

[8] V. Karlos, G. Solomos, and M. Larcher, "Analysis of the blast wave decay coefficient using the Kingery-Bulmash data," International Journal of Protective Structures, vol. 7, no. 3, pp. 409-429, 2016.

[9] M. Larcher, "Development of discrete cracks in concrete loaded by shock waves," International Journal for Impact Engineering, vol. 36, pp. 700-710, 2009.

[10] J. Li and H. Hao, "Internal and external pressure prediction of vented gas explosion in large rooms by using analytical and CFD methods," Journal of Loss Prevention in the Process Industries, vol. 49, pp. 367-381, 2017.

[11] EN14494, "Gas explosion venting protective systems," 2007.

[12] M. Larcher, F. Casadei, and G. Solomos, "Influence of venting areas on the air blast pressure inside tubular structures like railway carriages," Journal of Hazardous Materials, vol. 183, no. 1-3, pp. 839-846, 2010.

[13] P. J. Miller, "A reactive flow model with coupled reaction kinetics for detonation and combustion in non-ideal explosives," MRS Proceedings, vol. 418, 1995.

[14] M. Larcher, R. Forsberg, U. Bjoernstig, A. Holgersson, and G. Solomos, "Effectiveness of finite element modelling of damage and injuries for explosions inside trains," Journal of Transportation Safety and Security, vol. 8, pp. 83-100, 2015.

[15] G. Valsamos, F. Casadei, M. Larcher, and G. Solomos, "Implementation of flying debris fatal risk calculation in EUROPLEXUS,” Tech. Rep. JRC94805, Joint Research Centre, Publications Office of the European Union, Luxembourg, 2015.

[16] E. González Ferradás, F. Díaz Alonso, M. Doval Miñarro, A. Miñana Aznar, J. Ruiz Gimeno, and J. Sánchez Pérez, "Consequence analysis by means of characteristic curves to determine the damage to humans from bursting spherical vessels," Process Safety and Environmental Protection, vol. 86, no. 2, pp. 121-129, 2008. 
[17] S. Mannan, Lees' Loss Prevention in the Process Industries: Hazard Identification, Assessment and Control, Vol. 2, Elsevier, Amsterdam, 2005.

[18] Department of Defence USA, Unified Facilities Criteria, UFC 3-340-2, 2008.

[19] "NATO. STANAG 2280: design threat levels and handover procedure for temporary protective structures," 2008.

[20] G. F. Kinney and K. J. Graham, Explosive Shocks in Air, Springer, Berlin, Germany, 1985.

[21] D. Bogosian, J. Ferritto, and Y. Shi, "Measuring uncertainty and conservatism in simplified blast models," in Proceedings of 30th Explosive Safety Seminar, Atlanta, Georgia, August 2002.

[22] M. Larcher and F. Casadei, "Explosions in complex geometries-a comparison of several approaches," International Journal of Protective Structures, vol. 1, no. 2, pp. 169-195, 2010.

[23] M. Larcher, A. Stolz, L. Rüdiger, and M. Steyerer, "Statische Ersatzlasten und "Ingenieurverfahren", in Berichte aus dem Konstruktiven Ingenieurbau Workshop Bau-Protect, N. Gebbeken, K. Thoma, M. Rüdiger, and M. Klaus, Eds., pp. 117-130, Universität der Bundeswehr München, Neubiberg, Germany, 2012.

[24] FEMA, "Site and urban design for security-guidance against potential terrorist attacks," Tech. Rep. FEMA 430, FEMA, Washington, DC, USA, 2007.

[25] Joint Research Centre and Commissariat à l'énergie atomique et aux énergies alternatives, EUROPLEXUS, http://www-epx. cea.fr/.

[26] Commissariat à l'énergie atomique et aux énergies alternatives, CAST3M: http://www-cast3m.cea.fr/.

[27] ParaView: http://www.paraview.org.

[28] F. Daude and P. Galon, "On the computation of the baernunziato model using ALE formulation with HLL-and HLLCtype solvers towards fluid-structure interactions," Journal of Computational Physics, vol. 324, pp. 189-230, 2016.

[29] M. Larcher, F. Casadei, and G. Solomos, "Simulation of blast waves by using mapping technology in EUROPLEXUS," Tech. Rep. JRC91102, Joint Research Centre, Publications Office of the European Union, Luxembourg, 2014.

[30] E. Lee, J. Horning, and J. Kury, "Adiabatic expansion of high explosives detonation products," University of California, Lawrence Livermore National Laboratory, Livermore, CA, USA, 1968.

[31] B. Dobratz and P. Crawford, LLNL Explosives Handbook: Properties of Chemical Explosives and Explosive Simulants, University of California, Lawrence Livermore National Laboratory, Livermore, CA, USA, 1985. 


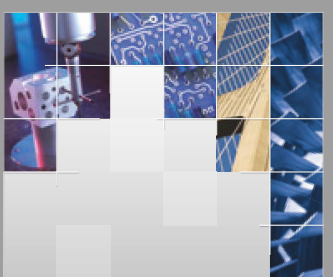

\section{Enfincering}
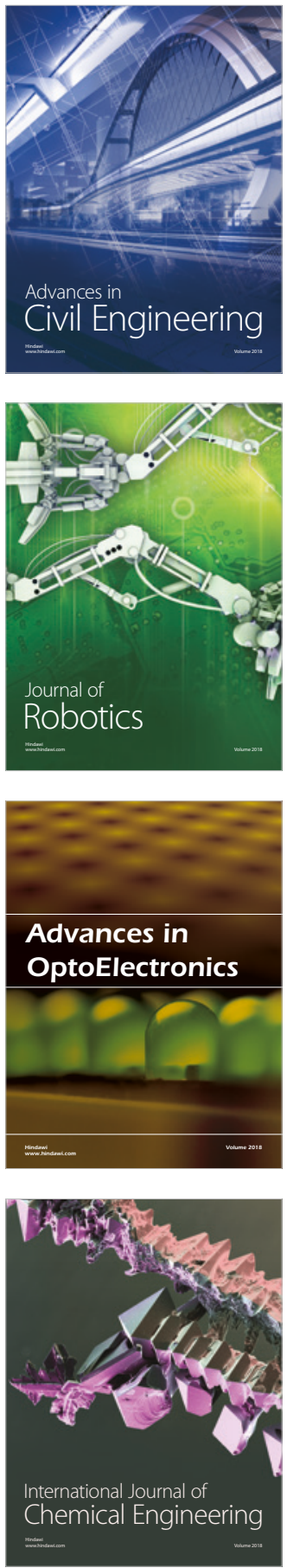

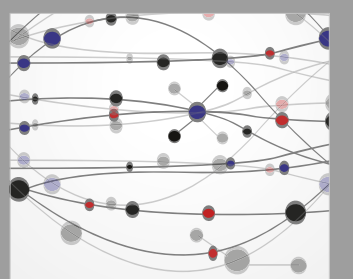

\section{Rotating \\ Machinery}

The Scientific World Journal

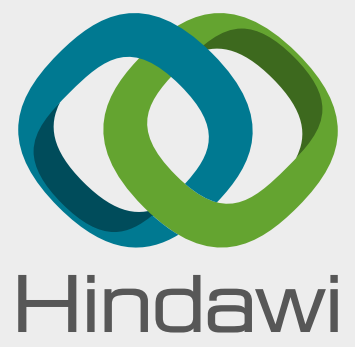

Submit your manuscripts at

www.hindawi.com
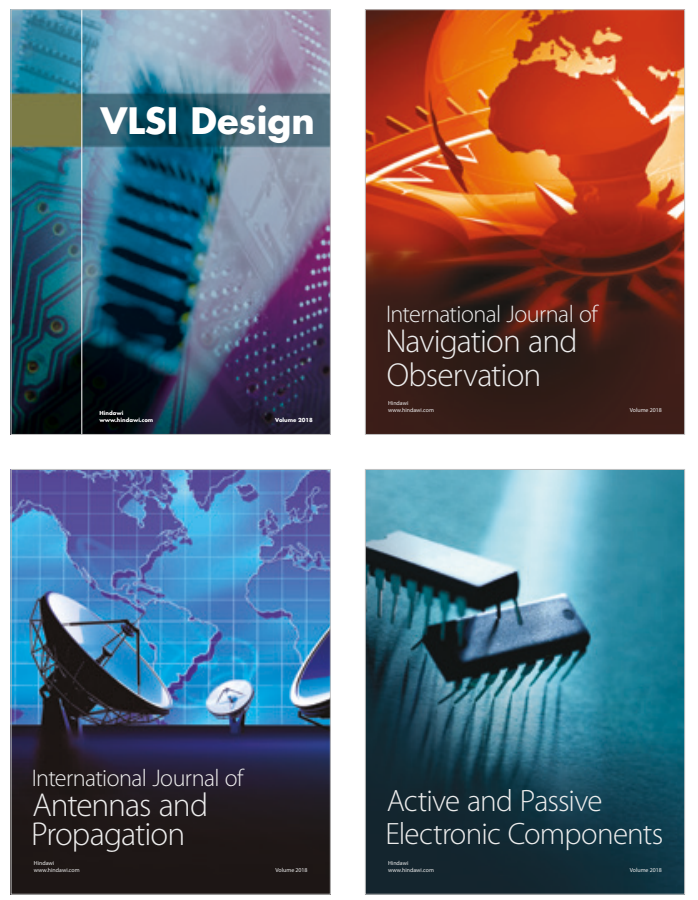
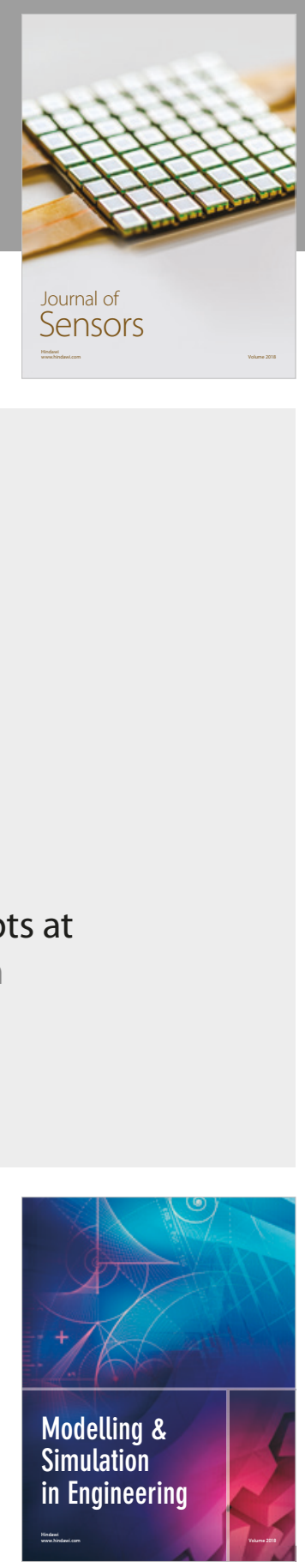

\section{Advances \\ Multimedia}
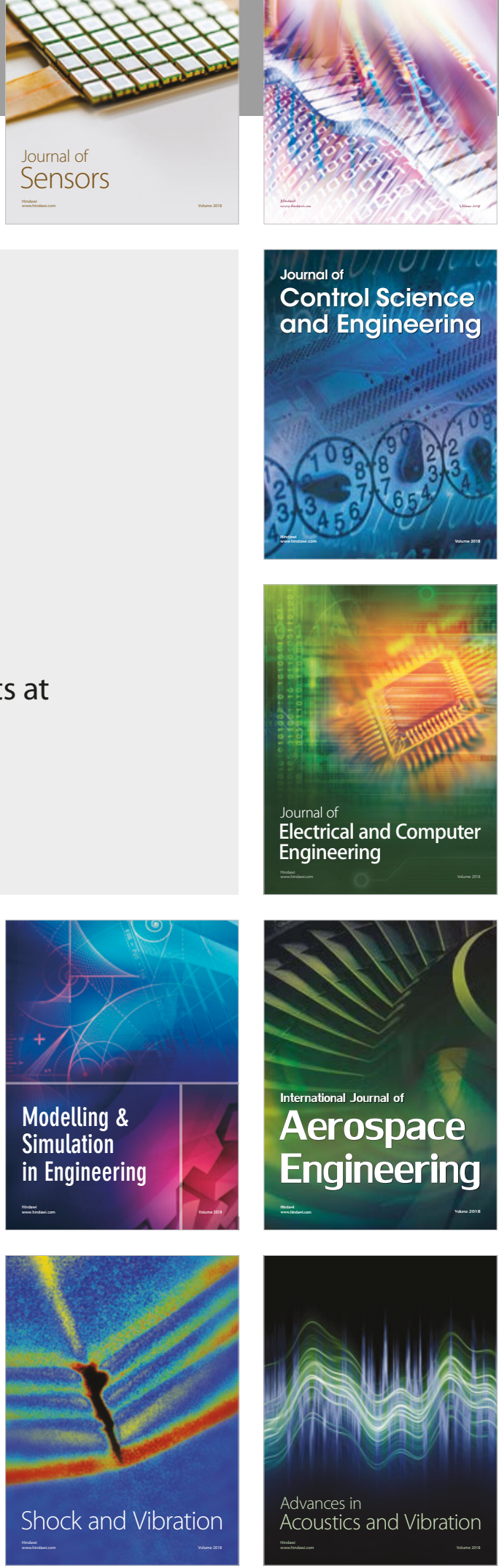